\title{
Clinical Trials Protocol and Data Management Shared Resource
}

National Cancer Institute

\section{Source}

National Cancer Institute. Clinical Trials Protocoland Data Management Shared

Resource. NCI Thesaurus. Code C39361.

The Clinical Trials Protocol and Data Management Shared Resource provides administrative support for Clinical Trials: assistance with protocol and budget development, submission and maintenance of IRB documentation, transmission of required information to study sponsors and federal oversight ag encies, coordination of IRB approval, protocol conduct and data collection with affiliated institutions, data retrieval and data transcription in compliance within the guidelines of GCP, quality assurance to ensure completeness and accuracy of data, maintenance of protocol and patient registration system, protocol monitoring and generation of reports, hiring/training of data managers, contract development and management. 